\title{
Transcriptome analysis of chicken kidney tissues following coronavirus avian infectious bronchitis virus infection
}

\author{
Feng Cong, Xiaoli Liu, Zongxi Han, Yuhao Shao, Xiangang Kong and Shengwang Liu*
}

\begin{abstract}
Background: Infectious bronchitis virus (IBV), a prototype of the Coronaviridae family, is an economically important causative agent of infectious bronchitis in chickens and causes an acute and highly contagious upper respiratory tract infections that may lead to nephritis. However, the molecular antiviral mechanisms of chickens to IBV infection remain poorly understood. In this study, we conducted global gene expression profiling of chicken kidney tissue after nephropathogenic IBV infection to better understand the interactions between host and virus.

Results: IBV infection contributed to differential expression of 1777 genes, of which 876 were up-regulated and 901 down-regulated in the kidney compared to those of control chickens and 103 associated with immune and inflammatory responses may play important roles in the host defense response during IBV infection. Twelve of the altered immune-related genes were confirmed by real-time RT-PCR. Gene ontology category, KEGG pathway, and gene interaction networks (STRING analysis) were analyzed to identify relationships among differentially expressed genes involved in signal transduction, cell adhesion, immune responses, apoptosis regulation, positive regulation of the I-kappaB kinase/NF-kappaB cascade and response to cytokine stimulus. Most of these genes were related and formed a large network, in which IL6, STAT1, MYD88, IRF1 and NFKB2 were key genes.
\end{abstract}

Conclusions: Our results provided comprehensive knowledge regarding the host transcriptional response to IBV infection in chicken kidney tissues, thereby providing insight into IBV pathogenesis, particularly the involvement of innate immune pathway genes associated with IBV infection.

Keywords: Infectious bronchitis virus, Kidney, Microarray, Transcriptome

\section{Background}

Avian infectious bronchitis virus (IBV) is a gamma coronavirus in the family Coronaviridae, which has been identified as the causative agent of infectious bronchitis (IB) as well as serious acute viral respiratory and urogenital diseases in commercial chickens flocks worldwide [1,2]. Infected chickens develop respiratory symptoms, kidney and oviduct lesions, reduced egg production with poor egg quality, and possible secondary complications $[3,4]$. IBV can replicate within the epithelial surfaces of the kidneys and cause granular degeneration, vacuolation, and desquamation of the tubular epithelium, and massive infiltration of heterophils in the interstitium. IBV-induced kidney

\footnotetext{
*Correspondence: swliu@hvriac.cn

Division of Avian Infectious Diseases, State Key Laboratory of Veterinary Biotechnology, Harbin Veterinary Research Institute, The Chinese Academy of Agricultural Sciences, Harbin 150001, The People's Republic of China
}

lesions are typically characterized by interstitial nephritis and tubule lesions that are most prominent in the medulla $[5,6]$.

Interactions between viruses and hosts occur at two levels: viral capacity to gain access to the target cell and competition between the viruses and host cells to control the cellular protein synthesis machinery. The virus/host interactions are largely determined by the virulence of the pathogen and the host immune response [7], and may lead to changes in host gene expression. However, many aspects of IBV-host interactions remain unclear. Elucidation of the basis of the interactions between IBV and kidney cells will provide new insights into the immune mechanisms underlying host antiviral strategies and the pathogenesis of viral infection. To date, a limited number of studies have examined host gene expression in response to IBV infection on a relatively large scale using 
microarrays or two-dimensional gel electrophoresis $[8,9]$. Gene expression alterations in avian embryonic tissues infected with IBV isolate B8358 were evaluated using a microarray containing 1191 unique chicken and turkey gene transcripts. Regulated expression has been established with several functional gene classes and pathways, including those coding kinases, interferon (IFN)-induced genes, chemokines, adhesins, vesicular trafficking and fusion proteins genes, extracellular matrix protein genes, the cell cycle, cell metabolism, physiology, and development, translation, RNA binding, lysosomal protein degradation and ubiquitination-related genes [10]. In addition, the gene transcription profiles of tracheal epithelial cells were examined 3 days postinfection with an attenuated IBV-Massachusetts strain [11]. These authors investigated 25 direct immune-related genes and found an upregulation in toll-like receptor (TLR)2, TLR3, IFN-induced antiviral genes $(\mathrm{Mx})$, and genes responsible for cytotoxic $\mathrm{T}$ cell killing, such as the Fas antigen and granzyme-A. More recently, changes in ex vivo and in ovo protein expression in Vero cells as well as chicken trachea and kidney tissues were reported after IBV infection $[8,9,12,13]$. However, there is limited information available regarding transcriptomics of host kidney cells in response to IBV infection. The current study was designed to compare the transcriptomes of kidney cells in IBV-infected chickens to those of control chickens. A subset of genes of interest identified by microarray analysis was validated by quantitative real-time reverse transcription polymerase chain reaction (qRT-PCR). The putative importance of some of these genes in IBV pathogenesis and immune response were also analyzed. The global gene expression profiling of chicken kidney tissue after nephropathogenic IBV infection will enable a better understanding of the pathogenesis of IBV infection and extend the knowledge of the nature of virus-host interactions.

\section{Methods}

Animals, viral infection and detection in kidney

One-day-old specific pathogen-free (SPF) chickens were obtained from the Laboratory Animal Center, Harbin Veterinary Research Institute, the Chinese Academy of Agricultural Sciences (Harbin, China). All animal experimental procedures were approved by the Ethical and Animal Welfare Committee of Heilongjiang Province, China.

Twenty 1-day-old SPF White Leghorn chicks were housed in separate isolators and divided into two groups of 10 chicks each. Each chick in group 1 was intranasally inoculated with $0.1 \mathrm{~mL}$ of $10^{5.5}$ median embryo infectious doses of strain ck/CH/LDL/091022 [14] at 15 days of age. Chicks in group 2 served as virus-free controls. Two birds in group 1 died at 5 and 6 days postinoculation (dpi), respectively, and fresh tissue samples of kidney from each dead bird were collected at the time of death. The kidneys from one bird in the control group were also collected at each time point corresponding to the bird deaths and then stored at $-80^{\circ} \mathrm{C}$ until further processed. Serum samples were collected at 12 days dpi from the remaining group 1 and control birds and assayed in triplicate using a commercial total antibody enzyme-linked immunosorbent assay (IDEXX Laboratories, Inc., Westbrook, MA, USA) according to the manufacturer's instructions. Serum-topositive ratios were calculated as described previously [15]. Individual serum titers were expressed as absorption values at an optical density at $630 \mathrm{~nm}$ according to the manufacturer's instructions. Real-time RT-PCR was used to quantify viral load in the kidneys of two dead IBV-infected chickens as described previously [16]. The remaining birds in both groups were killed humanely 15 dpi and kidney tissues were collected for virus recovery as described previously [15].

\section{RNA extraction, reverse transcription, RNA labeling, and cRNA hybridization}

Total RNA was extracted from kidneys of uninfected birds or those that died from IBV infection using TRIzol reagent (Invitrogen, Carlsbad, CA, USA) and then purified using the QiagenRNeasy Mini Kit (Qiagen, Valencia, CA, USA) according to the manufacturer's instructions. RNA was quantified and qualified using the Agilent 2100 Bioanalyzer (Agilent Technologies, Palo Alto, CA, USA). Briefly, $2 \mu \mathrm{g}$ of total RNA were converted to cDNA, synthesized to Cy3-labelled cRNA, and amplified for one round using a commercial array service (Agilent Technologies, America). The Cy3-labeled cRNA probes were hybridized to a $4 \times 44 \mathrm{~K}$ Agilent custom chicken oligo microarray (design ID: 017698). Scanning of the arrays was performed according to standard protocols using a G2505C Scanner (Agilent Technologies).

\section{Microarray analysis}

Microarray data were subjected to bioinformatic analysis to identify statistically significant changes in gene expression between samples using GeneSpring GX 11.0 software (Agilent Technologies). Annotations for the microarray genes were downloaded from the National Center for Biotechnology Information (http://www.ncbi.nlm.nih.gov/), the Gene Ontology (GO) (http://www.geneontology.org/), and UniProt (http://www.uniprot.org/) databases. The GO category (http://www.geneontology.org) based on biological process and KEGG (http://www.genome.jp/ kegg/) pathway analysis was determined for differentially expressed genes and a probability $(p)$-value $<0.05$ and a false discovery rate (FDR) $<0.05$ was used as a threshold. Gene interaction networks were analyzed using the STRING (http://string-db.org/) database of 
known and predicted protein interactions, which included direct (physical) and indirect (functional) associations.

\section{Real-time RT-PCR for confirmation}

Twelve genes of interest were selected for confirmation with $18 \mathrm{~S}$ serving as an endogenous control. Specific primers and probes were designed using Beacon Designer software 7.5 (Premier Biosoft International, Palo Alto, CA, USA). The primers and probes used for the RT-PCR assays are listed in Table 1. Total RNA was extracted using TRIzol Reagent (TaKaRa Biotech Co., Ltd., Dalian, China) according to the manufacturer's instructions. One-step real-time RT-PCR reactions were performed using the One Step PrimeScript ${ }^{\circ}$ RT-PCR kit (TaKaRa Biotech Co., Ltd.) on the Light-Cycler 480 real-time PCR system (Roche Diagnostics, Basel, Switzerland) according to the following steps: reverse transcription at $42^{\circ} \mathrm{C}$ for $10 \mathrm{~min}$, denaturation at $95^{\circ} \mathrm{C}$ for $10 \mathrm{~s}$ and 40 cycles at $95^{\circ} \mathrm{C}$ for $5 \mathrm{~s}$, $55^{\circ} \mathrm{C}$ for $20 \mathrm{~s}$, and $72^{\circ} \mathrm{C}$ for $10 \mathrm{~s}$, followed by a cooling step at $40^{\circ} \mathrm{C}$ for $10 \mathrm{~s}$. All samples were assayed in triplicate in each reaction. The data were analyzed using the standard curve method available with the LightCycler ${ }^{\circ}$ 480 Software ver. 1.5.

\section{Results}

\section{Clinical observations, serology, and IBV detection in} kidney tissues

The chicks inoculated with strain ck/CH/LDL/091022 showed clinical signs from dpi 3 to 13 . The diseased chicks were listless, huddled together, and displayed ruffled feathers and dark, shrunken combs. Two chicks died during the experiment and experienced obvious swelling and loss of blood to the kidneys, as well as distended tubules and ureters with urates, suggesting that $\mathrm{ck} / \mathrm{CH} /$ LDL/091022 was nephropathogenic (Figure 1) [14]. The clinical signs of the inoculated birds tended to gradually disappear after 13 dpi. No obvious clinical signs were observed in the uninfected control chickens during the experiment.

All chickens inoculated with strain ck/CH/LDL/091022 showed a positive serum antibody response at $12 \mathrm{dpi}$, whereas those in the control group showed a negative serum antibody response. IBV infection was also verified using real-time RT-PCR. Kidney tissue samples from each dead bird had detectable viral RNA with $3.26 \times 10^{8}$ and $1.32 \times 10^{8}$ copies/mg viral RNA, respectively. Viruses were not detected in the kidney samples from the control group. In addition, IBV was recovered from the kidneys of 5 out of 8 chickens challenged with strain ck/CH/LDL/091022 at 15 dpi using 9-day-old embryos; however, virus were not recovered in the kidneys of the eight control birds. Collectively, these results confirmed successful IBV infection of SPF chicken and those used for transcriptome analysis that died from IBV infection.

\section{Overview of differentially regulated genes after IBV infection}

RNA from kidney tissues retrieved from the two chickens that died at 5 and $6 \mathrm{dpi}$, respectively, and two uninfected control chickens were extracted and analyzed individually using a microarray to compare gene expression profiles between the groups. Microarray analysis identified 1777 genes, which were differentially regulated more than three-fold in response to IBV infection. Among the differentially expressed (DE) genes, 876 were up-regulated and 901 down-regulated. The DE genes were classified into 98 functional groups (see Additional file 1) according to the GO project for biological processes and the top 12 biological process groups of the genes are shown in Figure 2. The main GO categories for the upregulated genes were immune response (e.g., interleukin (IL)6 and IFN regulatory factor (IRF) 8), positive apoptosis regulation (e.g., BCL2-antagonist/killer 1 and Fas),

Table 1 Primers and probes used for the RT-PCR assays

\begin{tabular}{lllll}
\hline Gene & GenBank ID & Forward primers (5'-3') & Probes (5'-3') & Reverse primers (5'-3') \\
\hline STAT1 & NM_001012914 & AAGCAAACGTAATCTTCAGGATAAC & (FAM)-CAAGAAGACCCGATACACATGGCAA-(BHQ1) & TTTCTCTCCTCTTCAGACAGTTG \\
ISG12-2 & NM_001001296 & TTCCACTATCCAGTCTATCTCAATG & (FAM)-ACCTGCTCCTGGACCGATGCTTCT-(BHQ1) & GTGAATCTGTCTGTAAAGGATGAAC \\
SOCS3 & NM_204600 & ACTGCGCCCCAGGTGATG & (FAM)-CTCCCGGCAGCAGCACCCC-(BHQ1) & GGGAACTTGCTGTGGGTGAC \\
TNFAIP6 & NM_001037837 & GAGGCAGCGAGAAAATAGGTTC & (FAM)-CCATCCAGCCAGCAGCACACAC-(BHQ1) & GCTITACTATGGGGTAACCAACTC \\
IRG1 & NM_001030821 & TCCGAGATGTGGGCAAAGAC & (FAM)-TCGCTTGCTTCTCTGAATGACCACA-(BHQ1) & CCTACTCCAAGGGTATCCAGAATC \\
SPP1 & NM_204535 & CCAGAACAGCCGGACTTC & (FAM)-TGACATTCCTAGCAAGAGCCAAGAG-(BHQ1) & TGGAATCATTGTCATCATCATCATC \\
18S & FM165414 & GGTTGCAAAGCTGAAACTTAAAGG & (FAM)-ACTCCTGGTGGTGCCCTTCCGTCAA-(BHQ1) & TGAGGTTCCCGTGTTGAGTC \\
IFIT5 & XM_421662 & AAAAGAAGGCAAATCATGAGTACC & (FAM)-AATTCCTTGAAGACTCCCTGCTGC-(BHQ1) & TGATCCTCTATTGATTCTTCCAGAC \\
MX1 & NM_204609 & AGAAAGCAATAAGAAAAGCCCAAG & (FAM)-AATGCTACCATTAGTGCCAGCCAC-(BHQ1) & ACCAGATTTCAAGGGAAATTAGTC \\
OASL & NM_205041 & AGCACTGGTACAAGGAGATGTGG & (FAM)-CTGAAGTCCTCCCTGCCTGTGCCCT-(BHQ1) & CCAAGCAGCTCCAGCACAG \\
RSAD2 & XM_426208 & CTTAAGGAGGCGGGAATGGAG & (FAM)-TTGCTCACAATGCTGACGCTTGGC-(BHQ1) & TTGAACCACCGTTCCCTGATC \\
\hline
\end{tabular}




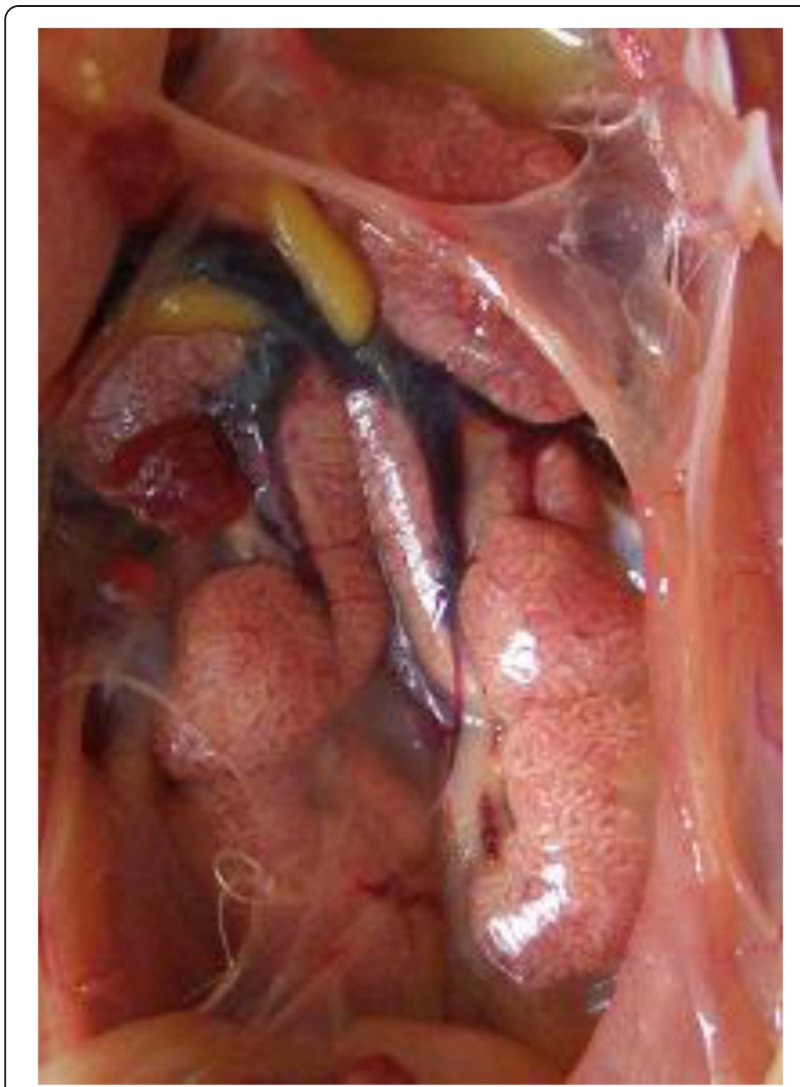

Figure 1 Renal lesions associated with IB caused by IBV strain ck/CH/LDL/091022. Note swollen kidneys with tubules and ureters distended with urates.

and negative apoptosis regulation (e.g., clusterin and microphthalmia-associated transcription factor), among others (Figure 2). The primary GO categories for the downregulated genes were response to cell adhesion (e.g., neuropilin 1 and contactin 1), signal transduction (e.g., endoglin and fibrinogen-like 2 ), and metabolic process (e.g., glutathione transferase and arylacetamidedeacetylaselike 4), among others (Figure 2). Particularly, the microarray results indicated that 103 genes associated with immune and inflammatory responses may play important roles in the host defense response during IBV infection (Table 2).

\section{Validation of microarray data by real-time RT-PCR}

Microarray analysis yields a large amount of data; therefore, it is important to validate differential expression by independent methods. Twelve immune and inflammatory response-related genes with significantly altered expression levels during IBV infection were selected for validation by real-time RT-PCR. The data demonstrated that the overall real-time RT-PCR results were consistent with those of the microarray analysis, although severalfold differences were observed between the two analytical methods because of intrinsic differences between the techniques. The real-time RT-PCR results revealed the same relative regulation pattern of transcription as those of the microarray data, thereby validating and confirming the microarray results, which indicated that the expression levels of many genes were significantly changed in response to IBV infection (Table 3).

\section{Pathway analysis}

To further define $D E$ gene function in chicken kidneys after IBV infection, the KEGG database was used to analyze pathways. The results showed that the DE genes were involved in the focal adhesion pathway, cytokinecytokine receptor interaction pathway, production of cell adhesion molecules, and peroxisome function as well as other pathways involved in host defense responses against IBV infection (Figure 3).

\section{STRING analysis of the relationships between DE genes}

The STRING database of known and predicted protein interactions was used to predict interactions of the DE genes listed in Table 2 (innate immune response, cytokinemediated signaling pathway, and inflammatory responserelated genes). Figure 4 summarizes the network of predicted associations for DE gene-encoded proteins. The results indicated that genes IL1RL1, IL1R1, IL-1 receptor-associated kinase 2, TLR4, signal transducer and activator of transcription 1 (STAT1), and myeloid differentiation primary response 88 (MYD88) were associated with many signaling pathways and other immune responses, whereas the genes IRF7, IFN-induced protein with tetratricopeptide repeats 1 (IFIT1), myxovirus resistance 1 (MX1), 2'-5' -oligoadenylate synthetase-like (OASL), IFN gamma receptor 1 (IFNGR1), and suppressor of cytokine signaling (SOCS)3 were also related. IL6, STAT1, MYD88, IRF1, and nuclear factor of kappa light polypeptide gene enhancer in B-cells 2 (NFKB2) were key in the interaction net and linked to SOCS1, SOCS3, STAT3, IRF1, chemokine (C-C motif) ligand (CCL)17, etc., which were further linked to many downstream genes, indicating that all of these genes were interrelated and formed a large network. However, not all genes were linked, indicating that their functions were either unrelated or have not yet been elucidated.

\section{Discussion}

Virulence may differ among IBV strains and, meanwhile, chickens showed various susceptibilities to an IBV strain [17]. To avoid discrepancies, a highly virulent nephropathogenic IBV strain, ck/CH/LDL/091022 [14], was used in this study to infect SPF chickens. Furthermore, fresh kidney tissue samples were collected and used for transcriptome analysis from a chicken infected with $\mathrm{ck} / \mathrm{CH} /$ LDL/091022 at the time of death. In addition, fresh kidney tissue samples were also collected from of a second 


\section{GO Biological Process}

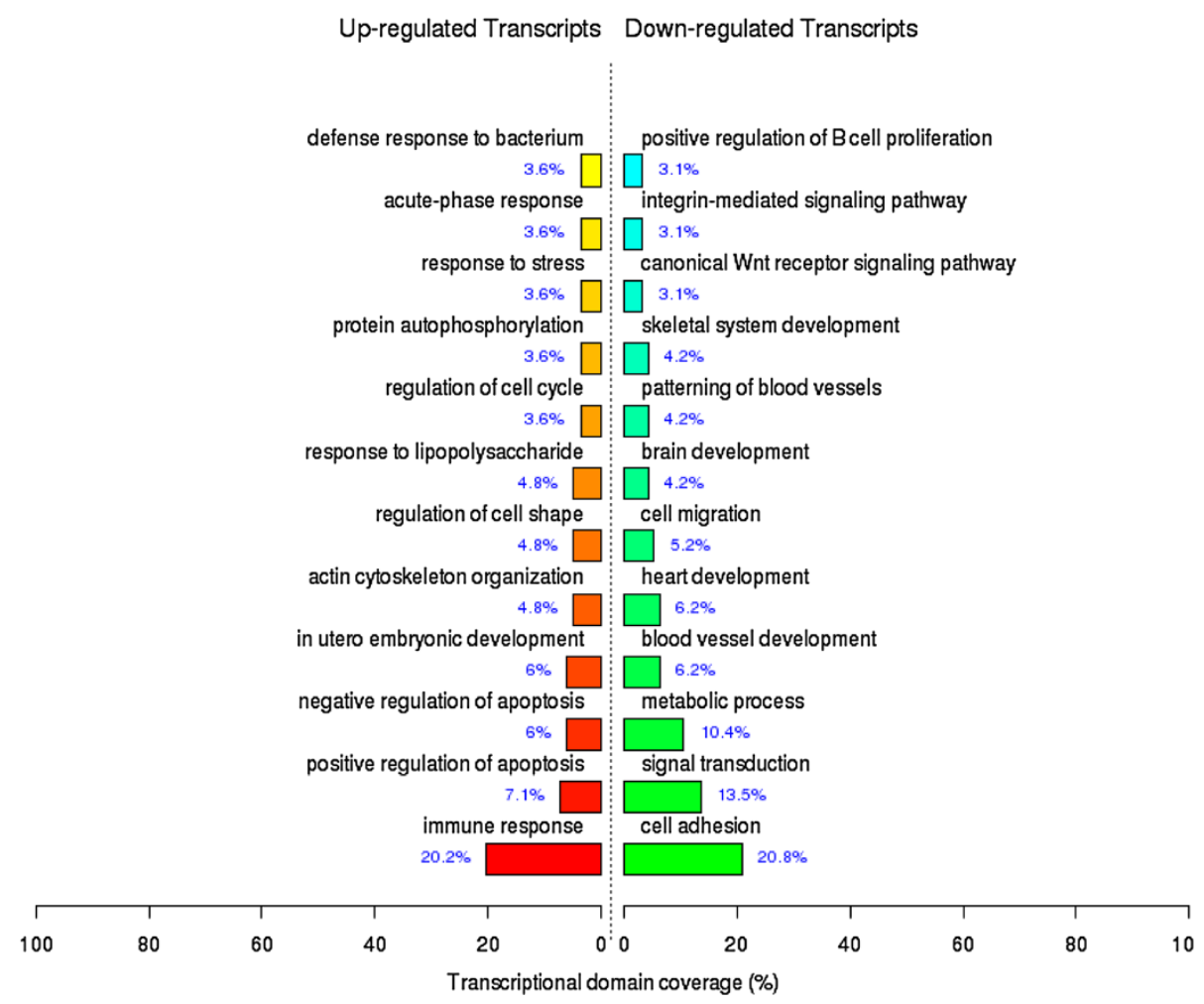

Figure 2 GO category based on biological processes for differentially expressed genes. The significant GO category for differentially expressed genes, the DE genes mainly clustered into 12 functional groups with varied numbers. $p$-value $<0.05$ and FDR $<0.05$ were used as a threshold to select significant GO categories. Exact $p$-value calculation for GO category in Additional file 1.

chicken infected with ck/CH/LDL/091022 at the time of death, processed individually, and used to confirm the results determined from the first chicken. Only genes that were differentially regulated more than three-fold in both IBV-infected chickens in response to IBV infection were compared to those of control birds and used for further analysis and validation by real-time PCR in this study.

The kidney is a primary target organ of nephropathogenic IBV, in which the transcriptional regulation of host genes after IBV infection can be used as a tool to obtain elaborate insight into virus-host interactions to unravel the pathogenic and/or immune mechanisms of IBV. To our knowledge, this is the first report to use microarray technology to acquire a global profile of host gene expression in chicken kidney cells after IBV infection. Herein, we identified 1777 genes that were differentially regulated more than three-fold compared to the corresponding control group and mostly associated with immune and inflammatory responses. Furthermore, 12 of the immune and inflammatory response DE genes were validated by qRT-PCR. Genes that were differentially expressed during infection can potentially provide insight into the complex regulatory phenomena in response to IBV infection. In our previous study, we identified expression alterations of 53 proteins in kidney tissues of chickens infected with another nephropathogenic IBV strain, ck/CH/LDL/97I, using two-dimensional gel electrophoresis $[8,9]$. The shared proteins/genes identified by the two methods were mainly involved in oxidoreductase, receptor binding, and transferase activities, whereas some were associated with immune responses. This might be at least due to the different sampling timepoints and the different virulence of viruses used for infecting chickens. The IBV strain ck/CH/LDL/ 091022 used in this study is highly nephropathogenic and more virulent than ck/CH/LDL/97I [14]. For Newcastle disease virus, it was reported that the more virulent strain persisted longer in the birds and, therefore, was able to increase the magnitude and duration of cell-mediated immunity [18].

Innate immunity provides a first line of defense against pathogens and can be rapidly activated following infection. Activation of the innate immune system relies on the recognition of pathogen-associated molecular patterns (PAMPs) by specific pattern-recognition receptors (PRRs) [19]. TLR4, TLR5, TLR15, and TLR16 belong to the TLR family and are involved in sensing and initiating 
Table 2 DE genes associated with immune and inflammatory responses

\begin{tabular}{|c|c|c|c|c|}
\hline Biological process & $\begin{array}{c}\text { Gene } \\
\text { symbol }\end{array}$ & Accession no & Gene description & $\begin{array}{c}\text { Fold } \\
\text { change }\end{array}$ \\
\hline \multirow[t]{42}{*}{ Innate immune response } & MALT1 & XM_413722 & mucosa associated lymphoid tissue lymphoma translocation gene 1 & +2.847583 \\
\hline & CLU & NM_204900 & clusterin & +9.196763 \\
\hline & DMBT1 & CR353989 & deleted in malignant brain tumors 1 & +2.036204 \\
\hline & DUSP4 & NM_204838 & dual specificity phosphatase 4 & +19.50851 \\
\hline & DUSP6 & NM_204354 & dual specificity phosphatase 6 & +2.1367393 \\
\hline & DUSP10 & NM_001031044 & dual specificity phosphatase 10 & +2.5124102 \\
\hline & LY96 & BX931181 & lymphocyte antigen 96 & +4.2568717 \\
\hline & SAMHD1 & NM_001030845 & SAM domain and HD domain 1 & +5.7296877 \\
\hline & ERAP1 & AJ851612 & endoplasmic reticulum aminopeptidase 1 & +5.731377 \\
\hline & HMGB1 & NM_204902 & high mobility group box 1 & +3.24355 \\
\hline & TMEM173 & CR354327 & transmembrane protein 173 & +9.81937 \\
\hline & CFI & XM_426329 & complement factor I & +5.3699164 \\
\hline & IL1R1 & NM_205485 & interleukin 1 receptor, type I & +2.814695 \\
\hline & IRAK2 & NM_001030605 & interleukin-1 receptor-associated kinase 2 & +2.1085246 \\
\hline & IRF7 & NM_205372 & interferon regulatory factor 7 & +4.334887 \\
\hline & JUN & NM_001031289 & jun proto-oncogene & +3.481312 \\
\hline & MAP3K14 & NM_001030927 & mitogen-activated protein kinase kinasekinase 14 & +2.0599 \\
\hline & MYD88 & NM_001030962 & myeloid differentiation primary response gene (88) & +2.0273855 \\
\hline & NCF2 & CR391316 & neutrophil cytosolic factor 2 & +9.767744 \\
\hline & NFKB2 & NM_204413 & $\begin{array}{l}\text { nuclear factor of kappa light polypeptide gene enhancer in B-cells } 2 \\
\qquad(\mathrm{p} 49 / \mathrm{p} 100)\end{array}$ & +4.372285 \\
\hline & MASP1 & NM_213586 & $\begin{array}{c}\text { mannan-binding lectin serine peptidase } 1 \text { (C4/C2 activating component } \\
\text { of Ra-reactive factor) }\end{array}$ & -5.984035 \\
\hline & PELI1 & NM_001012872 & pellino homolog 1 (Drosophila) & -2.6004448 \\
\hline & SIGIRR & NM_001199542 & single immunoglobulin and toll-interleukin 1 receptor (TIR) domain & -3.878401 \\
\hline & MRPS6 & NM_001031486 & mitochondrial ribosomal protein $\mathrm{S} 6$ & -7.8250012 \\
\hline & $\mathrm{IF|H} 1$ & NM_001193638 & interferon induced with helicase $\mathrm{C}$ domain 1 & +11.25022 \\
\hline & SRPK1 & XM_419265 & SFRS protein kinase 1 & +2.4798062 \\
\hline & TLR4 & NM_001030693 & toll-like receptor 4 & +2.019901 \\
\hline & TLR5 & CR353090 & toll-like receptor 5 & -2.4051092 \\
\hline & C1R & XM_416518 & complement component $1, \mathrm{r}$ subcomponent & +13.635473 \\
\hline & C1S & NM_001030777 & complement component 1 , s subcomponent & +16.500757 \\
\hline & C3 & NM_205405 & complement component 3 & +25.31715 \\
\hline & C4BPA & NM_204664 & complement component 4 binding protein, alpha & -2.476029 \\
\hline & $\mathrm{C} 8 \mathrm{~B}$ & BX934795 & complement component 8 , beta polypeptide & -2.8326783 \\
\hline & TRIM25 & XM_415653 & tripartite motif-containing 25 & +3.5009384 \\
\hline & COLEC12 & NM_001039599 & collectin sub-family member 12 & -11.011364 \\
\hline & $\mathrm{ITCH}$ & XM_417330 & itchy homolog E3 ubiquitin protein ligase (mouse) & +2.3843937 \\
\hline & MARCO & NM_204736 & macrophage receptor with collagenous structure & -3.0318317 \\
\hline & FADD & XM_421073 & Fas (TNFRSF6)-associated via death domain & -2.127713 \\
\hline & IL18R1 & NM_001145225 & interleukin 18 receptor 1 & +3.5603435 \\
\hline & VNN1 & NM_001039288 & vanin 1 & +30.900112 \\
\hline & IL1RL1 & NM_204275 & interleukin 1 receptor-like 1 & -9.989544 \\
\hline & ADAR & AM179858 & adenosine deaminase, RNA-specific & +2.2560015 \\
\hline
\end{tabular}


Table 2 DE genes associated with immune and inflammatory responses (Continued)

\begin{tabular}{|c|c|c|c|c|}
\hline \multirow{27}{*}{$\begin{array}{l}\text { Cytokine-mediated signaling } \\
\text { pathway }\end{array}$} & USP18 & CR354286 & ubiquitin specific peptidase 18 & +14.18105 \\
\hline & EGR1 & CR389000 & early growth response 1 & +4.516151 \\
\hline & IRF8 & NM_205416 & interferon regulatory factor 8 & +4.638908 \\
\hline & IFI35 & BX934680 & interferon-induced protein 35 & +4.3734093 \\
\hline & IFNGR1 & NM_001130387 & interferon gamma receptor 1 & +2.4954834 \\
\hline & IL6 & NM_204628 & interleukin 6 (interferon, beta 2) & +75.02113 \\
\hline & IL13RA2 & NM_001048078 & interleukin 13 receptor, alpha 2 & +5.0171814 \\
\hline & IRF1 & NM_205415 & interferon regulatory factor 1 & +7.012157 \\
\hline & IRF7 & NM_205372 & interferon regulatory factor 7 & +4.334887 \\
\hline & LIFR & NM_204575 & leukemia inhibitory factor receptor alpha & +2.3569908 \\
\hline & $M \times 1$ & NM_204609 & $\begin{array}{l}\text { myxovirus (influenza virus) resistance 1, interferon-inducible protein } \\
\qquad \text { p78 (mouse) }\end{array}$ & +48.569965 \\
\hline & NCAM1 & NM_001242604 & neural cell adhesion molecule 1 & -7.5168643 \\
\hline & IP6K2 & NM_001030596 & inositol hexakisphosphate kinase 2 & +2.4397569 \\
\hline & PML & XM_413690 & promyelocytic leukemia & +3.3186321 \\
\hline & $\mathrm{ZC} 3 \mathrm{H} 15$ & NM_001006510 & zinc finger $\mathrm{CCCH}$-type containing 15 & -2.916313 \\
\hline & B2M & NM_001001750 & beta-2-microglobulin & +6.515671 \\
\hline & PTPN1 & L20630 & protein tyrosine phosphatase, non-receptor type 1 & +3.4228158 \\
\hline & PTPN2 & NM_001199387 & protein tyrosine phosphatase, non-receptor type 2 & +2.9723573 \\
\hline & CX3CL1 & NM_001077232 & chemokine (C-X3-C motif) ligand 1 & +10.652483 \\
\hline & STAT1 & NM_001012914 & signal transducer and activator of transcription 1, $91 \mathrm{kDa}$ & +12.110944 \\
\hline & STAT3 & NM_001030931 & $\begin{array}{c}\text { signal transducer and activator of transcription } 3 \text { (acute-phase } \\
\text { response factor) }\end{array}$ & +3.900449 \\
\hline & VCAM1 & BX950651 & vascular cell adhesion molecule 1 & -2.8632648 \\
\hline & OASL & NM_205041 & 2'-5'-oligoadenylate synthetase-like & +101.55924 \\
\hline & SOCS1 & NM_001137648 & suppressor of cytokine signaling 1 & +15.976115 \\
\hline & sOCS3 & NM_204600 & suppressor of cytokine signaling 3 & +30.325502 \\
\hline & CD44 & NM_204860 & CD44 molecule (Indian blood group) & +5.446098 \\
\hline & CD74 & NM_001001613 & $\begin{array}{c}\text { CD74 molecule, major histocompatibility complex, class II } \\
\text { invariant chain }\end{array}$ & -7.0005507 \\
\hline \multirow[t]{16}{*}{ Inflammatory response } & CDO1 & CR353781 & cysteine dioxygenase, type I & -6.4660363 \\
\hline & CEBPB & NM_205253 & CCAAT/enhancer binding protein (C/EBP), beta & +3.5429432 \\
\hline & IL23R & XM_422533 & interleukin 23 receptor & +3.9536817 \\
\hline & $\mathrm{EPHX} 2$ & NM_001033645 & epoxide hydrolase 2, cytoplasmic & -2.0498822 \\
\hline & LY96 & BX931181 & lymphocyte antigen 96 & +4.2568717 \\
\hline & BLNK & NM_204908 & B-cell linker & +3.781344 \\
\hline & $\mathrm{AOAH}$ & XM_418835 & acyloxyacyl hydrolase (neutrophil) & +3.389245 \\
\hline & $\mathrm{AOX} 1$ & NM_001038692 & aldehyde oxidase 1 & -3.0358417 \\
\hline & IGFBP4 & NM_204353 & insulin-like growth factor binding protein 4 & -2.5591307 \\
\hline & IL6 & NM_204628 & interleukin 6 (interferon, beta 2) & +75.02113 \\
\hline & IRAK2 & NM_001030605 & interleukin-1 receptor-associated kinase 2 & +2.1085246 \\
\hline & KNG1 & XM_422766 & kininogen 1 & -2.1926415 \\
\hline & LIPA & AJ719682 & lipase A, lysosomal acid, cholesterol esterase (Wolman disease) & +3.7113423 \\
\hline & LY75 & NM_001037836 & lymphocyte antigen 75 & +3.9913418 \\
\hline & NGFB & M26810 & nerve growth factor, beta polypeptide & -2.035398 \\
\hline & NOX4 & NM_001101829 & NADPH oxidase 4 & -9.4226265 \\
\hline
\end{tabular}


Table 2 DE genes associated with immune and inflammatory responses (Continued)

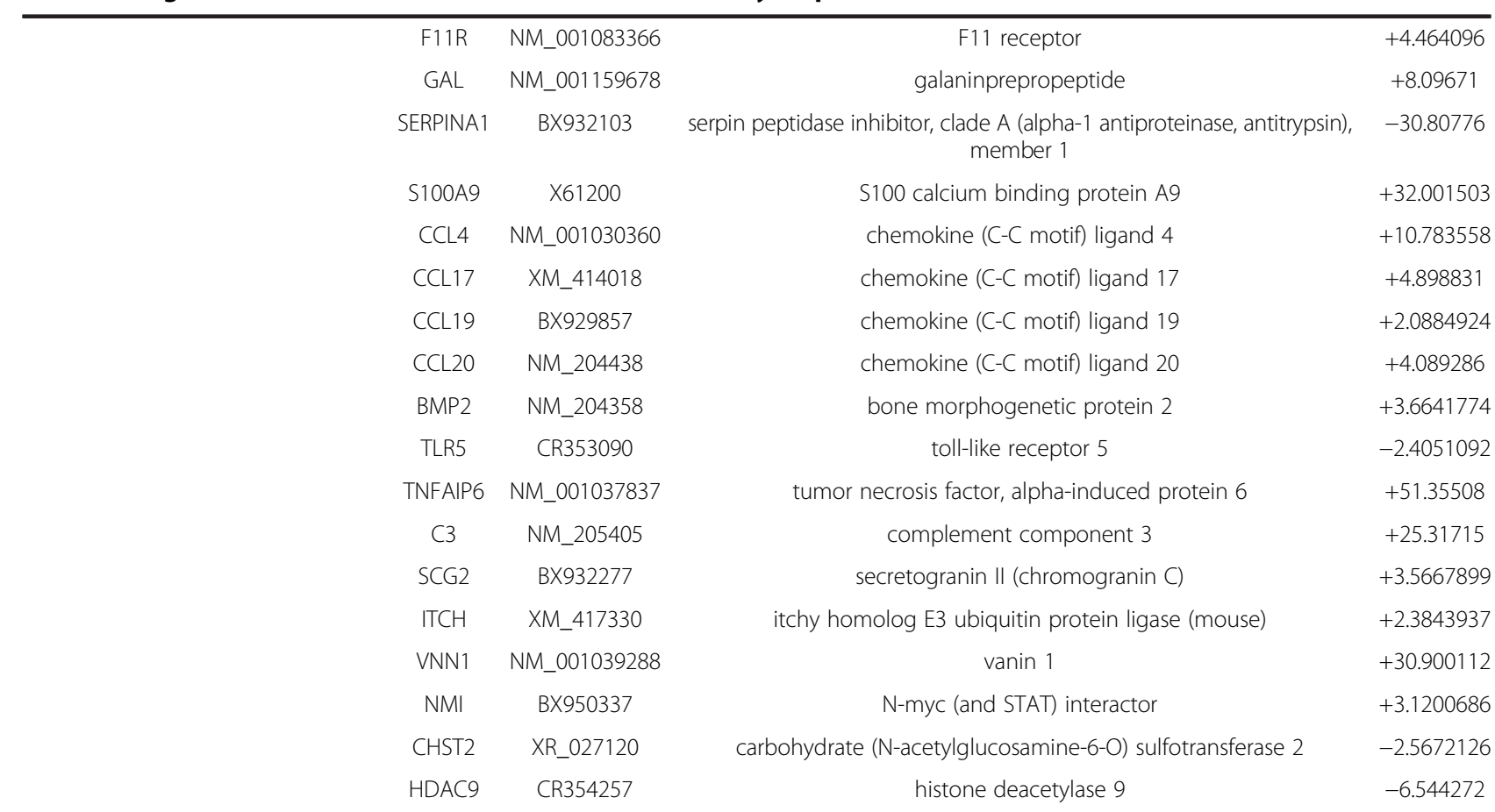

Genes were considered significantly up-regulated or down-regulated if the change in their relative expression levels was $\geq 2$-fold or $\leq-2$-fold.

The DE genes associated with innate immune response, cytokine-mediated signaling pathway, and inflammatory responses were assigned based on GO term. Most genes could also be classified into other categories, but only innate immune response, cytokine-mediated signaling pathway, and inflammatory responses were considered.

+: up-regulated;-: down-regulated.

immune responses to viral infection [20]. All of the TLRs were significantly up-regulated after IBV infection. Reportedly, severe acute respiratory syndrome (SARS-CoV) and mouse hepatitis virus (MHV) infection can also induce TLR4 expression [21], suggesting that TLR4

Table 3 Validation of microarray data by qRT-PCR

\begin{tabular}{llll}
\hline Genes & Accession no & $\begin{array}{l}\text { Microarray fold } \\
\text { change } \\
\text { (infected/control) }\end{array}$ & $\begin{array}{l}\text { Real-time RT-PCR } \\
\text { fold change } \\
\text { (infected/control) }\end{array}$ \\
\hline ISG12-2 & NM_001001296 & +141.21 & +197.36 \\
SPP1 & NM_204535 & +19.54 & +3.66 \\
IRG1 & NM_001030821 & +21.26 & +4.28 \\
STAT1 & NM_001012914 & +16.57 & +14.60 \\
TNFAIP6 & NM_001037837 & +136.96 & +3.33 \\
IFITM3 & XM_420925 & +422.64 & +234.00 \\
SOCS3 & NM_204600 & +24.75 & +9.86 \\
IL6 & NM_204628 & +97.97 & +490.15 \\
IFIT5 & XM_421662 & +130.57 & +84.17 \\
RSAD2 & XM_426208 & +165.91 & +193.13 \\
MX1 & NM_204609 & +91.194 & +16.60 \\
OASL & NM_205041 & +185.05 & +22.07 \\
\hline
\end{tabular}

qRT-PCR results of 12 immune and inflammatory response related genes in kidney tissues from IBV-infected chickens compared to those of control chickens. qRT-PCR levels of RNA for a given gene were normalized against the 18S RNA.

" + " indicates up-regulated genes. possibly has a similar effect both in avian and mammalian species as well as during coronavirus infection. Similar to the present results, a previous study reported that the transcription level of TLR15, a poultry-specific TLR, was significantly up-regulated after infection with Marek's disease virus [22], infectious bursa disease virus infection [23], and avian influenza virus H9N2-infected chickens in the lungs [24], implicating a similar role of TLR15 in sensing and initiating responses to viruses after infection.

Melanoma differentiation-associated gene-5 (MDA5 or IFIH1) belongs to the retinoic acid-inducible-like helicase family of PRRs and sense viral RNA in the cytoplasm. In this study, MDA5 expression levels were significantly increased after IBV infection. SARS-Cov infection can also induce MDA5 expression in vitro and MHV is recognized by MDA5 in brain macrophages, oligodendrocytes, and microglial cells [25-27], suggesting a role of chicken MDA5 against IBV infection and a possible correlation with induction of the inflammatory response.

Although distinct microbial PAMPs activate different TLRs, they ultimately cross paths during the transcriptional activation of IRF3, IRF7, and NF-kB, all of which translocate to the nucleus and activate transcription of type I IFN (IFN- $\alpha$ and IFN- $\beta$ ) [28] and the subsequent IFN-enhanced production of IFN-stimulated genes 


\section{KEGG}

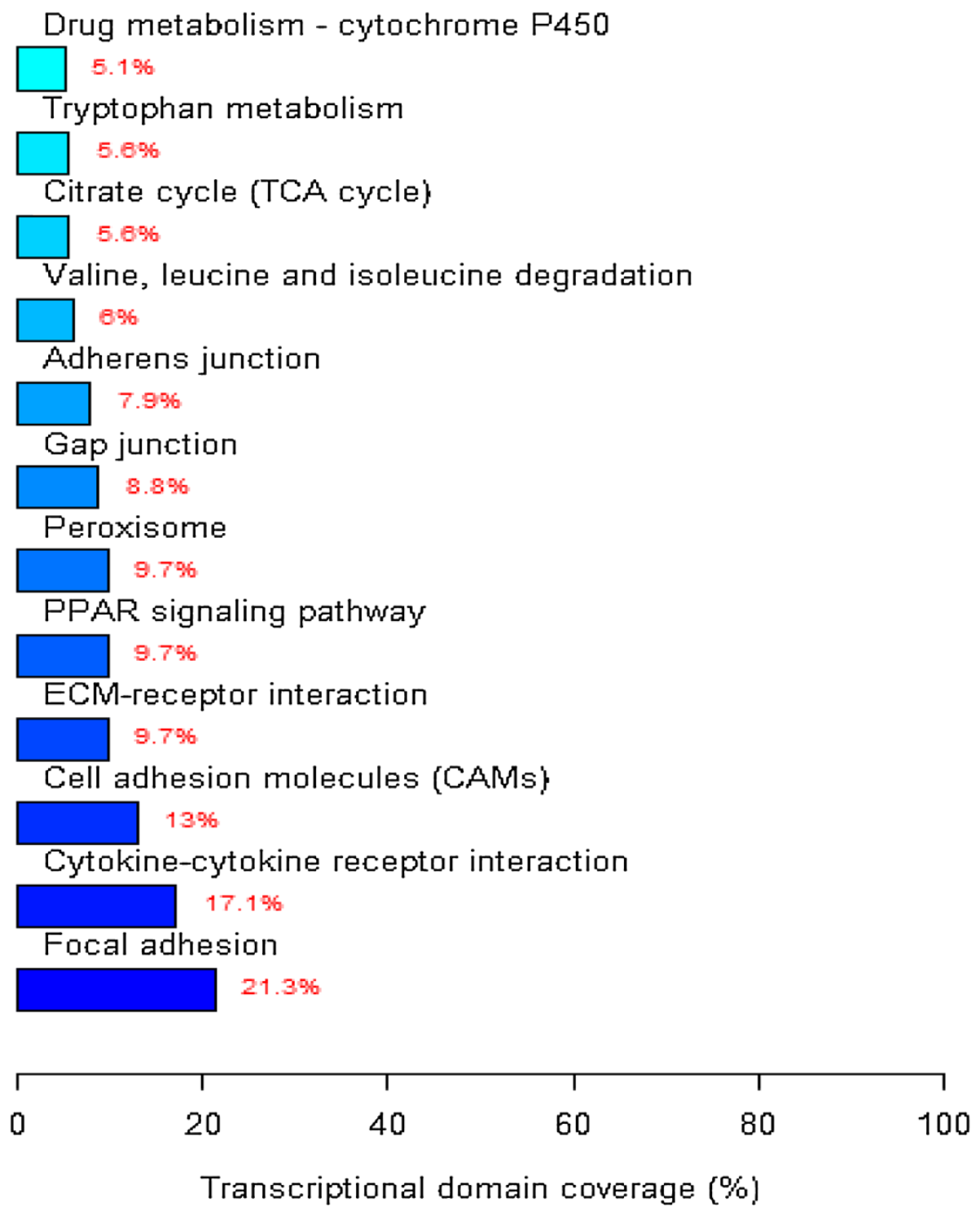

Figure 3 KEGG pathway analysis for differentially expressed genes. The significant pathway for differentially expressed genes. A $p$-value $<0.05$ and FDR $<0.05$ were used as a threshold to select significant KEGG pathways. Exact $p$-value calculation for KEGG pathway analysis in Additional file 2.

(ISGs). IBV can induce acute IFN- $\alpha$ production [29] and the ability to induce IFN is linked to the virulence and adaptability of the IBV strain to a particular host system [30,31]. In this study, IFN expression was not significantly upregulated after IBV infection; however, some IRFs and ISGs, such as IRF1, IRF7, IRF8, IRF10, NF-kB, STAT1, MyD88, OSAL, MX1, IFIT5, ISG12-2, RSAD2, IFI35, and IFI27L2 were all up-regulated. Therefore, we hypothesized that IFN might be induced during early infection, as indicated by the subsequent enhanced production of ISGs because increased expression of a variety of ISGs, such as OSAL, MX1, IFIT5, ISG12-2, RSAD2, IFI35, protein kinase R (PKR), and IFI27L2, was found in this study. STAT proteins form homodimeric and heterodimeric complexes to activate transcription of some ISGs [32]. Similar to the results in the current study, a previous report showed that STAT1 expression was significantly increased response to IBV infection in ovo [10], suggesting that IBV infection can activate the JAK-STAT pathway and activate transcription of ISGs. MX1 is a dynamin-like large guanosinetriphosphatase (GTPase), which has antiviral activity against a wide range of RNA viruses [33]. PKR (also known as EIF2 $\alpha$ K2) is constitutively expressed as an inactive kinase that is activated by 


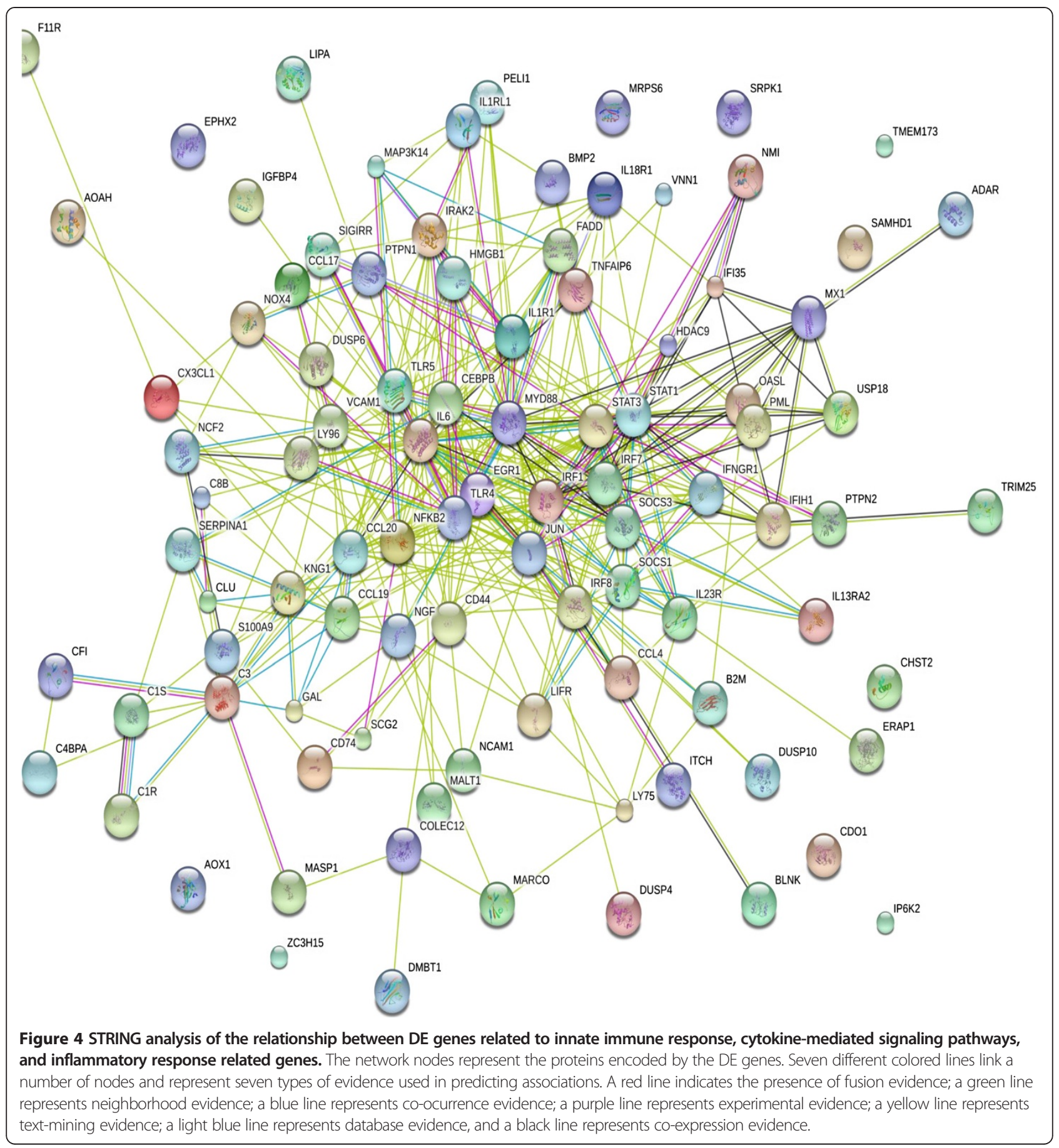

viral double-stranded RNA and plays an important role in the cellular antiviral response pathway. OASL is a member of the OAS gene family, which in combination with RNaseL constitutes an antiviral RNA decay pathway [34]. The activation of both OAS and PKR results in global degradation of cellular RNA and translation inhibition, which may also inhibit viral propagation
[28]. In addition, we found that the MxGTPase pathway, the protein kinase $\mathrm{R}$ pathway, and the $2^{\prime}-5^{\prime}$ oligoadenylatesynthetase-directed ribonuclease $\mathrm{L}$ pathway might be involved in anti-IBV infection, but the ISG15 ubiquitin-like pathway was not. These pathways might exert their effects through different mechanisms of action, such as direct targeting of viral entry, inhibition of protein synthesis, 
or degradation of viral RNA [35], and play an important role in anti-IBV responses and host defense during IBV infection.

Viral infections induce a pro-inflammatory response that include cytokine and chemokine expression [36]. In this study, increased expression of IL6, IL18, IL10RA, IL17RA, CCL4, CCL20, CCL17, and CCL19 was observed after IBV infection; however, chemokine (C-X-C motif) ligand 12 expression was found to be decreased. IL6 was also up-regulated during other nidovirus infections, such as SARS-CoV, porcine respiratory coronavirus, equine arteritis virus, and transmissible gastroenteritis coronavirus (TGEV) [37-39]. These findings suggested that IL6, IL18, and IL10 may have similar functions in IBV infection as those in other coronavirus infections. The important negative regulators SOCS-1 and SOCS-3 were significantly up-regulated in this study. SOCS-1 and SOCS-3 participate in the feedback system of IL6 signal transduction by binding to phosphorylated tyrosine residues of a component of the IL6 receptor gp130 [40]. The observed changes in expression of IL6, SOCS-1, and SOCS-3 in kidney tissues of IBV-infected chickens suggested a balance between pro-and anti-inflammatory cytokines, which seemed to be critical for IBV pathogenesis.

Our data also presented an interesting set of apoptosisrelated genes after IBV infection. In this study, the expression of the Fas, caspase (casp) 1, casp9, casp18, Bcl-2L1, BCL2-antagonist/killer 1, myeloid cell leukemia sequence 1 (Mcl-1), and eukaryotic initiation factor 2a genes was significantly increased compared to the control group. The caspase proteins are executioners of apoptosis [41]. Extrinsic signals to initiate the apoptotic pathways from receptors (e.g., Fas ligand) culminate in the activation of caspase 8 , which activates the effector caspase 3 , while intrinsic signaling requires the participation of the mitochondria in releasing cytochrome $\mathrm{c}$ to activate caspase 9 for the downstream activation of caspase 3. Both proapoptotic (e.g., Bax and Bak) and anti-apoptotic proteins (e.g., Bcl-2 and Bcl-XL) from the Bcl-2 family are key proteins in the intrinsic apoptosis signaling pathway [28]. Recent reports have suggested that IBV also triggers apoptosis during the late stages of its cytolytic infection cycle. Similar results were found in IBV-infected Vero cells at $24 \mathrm{~h}$ postinfection, revealing an up-regulation at the transcriptional level of both pro-apoptotic Bak and pro-survival Mcl-1, which may play essential roles in maintaining the intricate balance between life and death of infected cells to ensure a successful infection cycle [42]. Taken together, it can be hypothesized that IBV activates apoptosis through Fas/FasL activation and mitochondrial-dependent pathways, similar to that of TGEV $[43,44]$. Furthermore, Bak and Mcl-1 may play key roles in the regulation of IBV-induced apoptosis, not only at an early stage of infection in vitro, but also at a late stage of infection in vivo. In other words, apoptosis is a nonspecific defense mechanism against IBV infection through abortion of viral multiplication by premature lysis of infected cells.

In addition, several other immune function-related genes were up-regulated, including major histocompatibility complex (MHC) class I antigen, MHC BF2 class I, MR1, MHC BF1 class I, immunoresponsive 1 homolog, secreted phosphoprotein 1 , the complement system $(\mathrm{C} 3, \mathrm{C} 1 \mathrm{~S}, \mathrm{C} 4$ and $\mathrm{C} 1 \mathrm{R})$, among other genes involved in the IFR response (IRF1,IRF7,IRF8, IFIH1, IFIT-like ISG12-2, and IFITM3 ). The above results indicated that both adaptive and innate immunity responded to IBV infection and the complement system might be a functional bridge between innate and adaptive immune responses to allow an integrated host defense to pathogenic challenges [45].

The data in this study were obtained from kidney of chickens infected with a nephropathogenic IBV strain at 5 and $6 \mathrm{dpi}$, respectively, concomitant with the peak of the virus titers in the kidney [14]. The analysis of the global profile of host gene expression provided a good overview of host response to IBV infection and is helpful for understanding IBV mechanism of disease or death. The results also provided basic information needed to extend our understanding of the nature of the virus-host interactions. However, analysis of the innate host immune response in kidneys at those time points may not be sufficient to completely understand IBV immunologic mechanisms. In addition, the roles of individual cell types in this response cannot be effectively measured in this way. Therefore, further analysis of the above-mentioned concerns is warranted. Given the consistent utilization of current vaccines and the continued emergence of new virulent IBV genotypes throughout the world, the development of better vaccines and control strategies will require a greater understanding of pathogenic mechanisms.

\section{Conclusions}

We screened and identified differentially expressed transcripts in IBV-infected chicken kidney tissues using microarray analysis. A total of $103 \mathrm{DE}$ genes were identified in this study, which were associated with immune and inflammatory responses, as well as the cytokine-mediated signaling pathway; therefore, these genes may play major roles in the host defense response or/and IBV pathogenesis. Combining network analysis with differential gene expression analysis helped to uncover high-confidence genes, such as IL6, TLR4, STAT1, MYD88, IRF1, and NFKB2, and several immune-related genes, such as IRF7, IFIT1, MX1, OASL, IFNGR1, and SOCS3, that have potentially important consequences to IBV infection. Our results should prove helpful to elucidate the pathogenic mechanisms of IBV and the host antiviral response. 


\section{Additional files}

\section{Additional file 1: The complete microarray dataset of the DE genes} with GO biological process annotations.

Additional file 2: The $p$-value of KEGG pathway analysis.

\section{Abbreviations}

IBV: Infectious bronchitis virus; IB: Infectious bronchitis; TLR: Toll-like receptors; Mx: IFN-induced antiviral genes; SPF: Specific pathogen free; Dpi: Day post infection; qRT-PCR: Quantitative real-time RT-PCR: DE: Differential expression; PRRs: Pattern recognition receptors; PAMPs: Pathogen associated molecular patterns; MHV: Mouse hepatitis virus; MDA5: Melanoma differentiation-associated gene-5; ISGs: IFN-stimulated genes; TGEV: Transmissible gastroenteritis coronavirus.

\section{Competing interests}

The authors declare that they have no competing interests.

\section{Authors' contributions}

$\mathrm{SL}$ designed the study. SL and FC drafted the manuscript. $F C, X L, Z H$, and $Y S$ performed virus infection and tests for the presence of IBV. FC made substantial contributions to the bioinformatics and statistical analysis. FC and $X L$ confirmed differential gene expression by real-time RT-PCR. SL wrote the manuscript. SL and XK revised the manuscript. All authors read and approved the final manuscript.

\section{Acknowledgements}

This work was supported by grants from the China Agriculture Research Systerm (No. CARS-41-K12) and Special Fund for Agro-scientific Research in the Public Interest (No. 201203056).

Received: 23 April 2013 Accepted: 26 October 2013

Published: 30 October 2013

\section{References}

1. Carstens E: Report from the 40th meeting of the executive committee of the international committee of taxonomy of viruses. Arch Virol 2009, 154:1571-4.

2. Kinde H, Daft BM, Castro AE, Bickford AA, Gelb J Jr, Reynolds B: Viral pathogenesis of a nephrotropic infectious bronchitis virus isolated from commercial pullets. Avian Dis 1991, 35:415-21.

3. Ahmed Z, Naeem K, Hameed A: Detection and seroprevalence of infectious bronchitis virus strains in commercial poultry in Pakistan. Poult Sci 2007, 86:1329-35.

4. Liu S, Kong X: A new genotype of nephropathogenic infectious bronchitis virus circulating in vaccinated and non-vaccinated flocks in China. Avian Pathol 2004, 33:321-7.

5. Han Z, Sun C, Yan B, Zhang X, Wang Y, Li C, Zhang Q, Ma Y, Shao Y, Liu Q, Kong $X$, Liu S: A 15-year analysis of molecular epidemiology of avian infectious bronchitis coronavirus in China. Infect Genet Evol 2011. 11:190-200

6. Mahgoub K, Bassiouni A, Afify MA, Rabie SN: The prevalence of infectious bronchitis (IB) outbreaks in some chicken farms III: cross protection of vaccinated chickens versus field IB virus. J Am Sci 2010, 6:94-108.

7. Schneider RJ, Shenk T: Impact of virus infection on host cell protein synthesis. Annu Rev Biochem 1987, 56:317-32.

8. Cao Z, Han Z, Shao Y, Geng H, Kong X, Liu S: Proteomic analysis of chicken embryonic trachea and kidney tissues after infection in ovo by avian infectious bronchitis coronavirus. Proteome Sci 2011, 9:11.

9. Cao Z, Han Z, Shao Y, Liu X, Sun J, Yu D, Kong X, Liu S: Proteomics analysis of differentially expressed proteins in chicken trachea and kidney after infection with the highly virulent and attenuated coronavirus infectious bronchitis virus in vivo. Proteome Sci 2012, 10:24.

10. Dar A, Munir S, Vishwanathan S, Manuja A, Griebel P, Tikoo S, Townsend H, Potter A, Kapur V, Babiuk LA: Transcriptional analysis of avian embryonic tissues following infection with avian infectious bronchitis virus. Virus Res 2005, 110:41-55

11. Wang X, Rosa AJ, Oliverira HN, Rosa GJ, Guo X, Travnicek M, Girshick T: Transcriptome of local innate and adaptive immunity during early phase of infectious bronchitis viral infection. Viral Immunol 2006, 19:768-74.
12. Emmott E, Rodgers MA, Macdonald A, McCrory S, Ajuh P, Hiscox JA: Quantitative proteomics using stable isotope labeling with amino acids in cell culture reveals changes in the cytoplasmic, nuclear, and nucleolar proteomes in Vero cells infected with the coronavirus infectious bronchitis virus. Mol Cell Proteomics 2010, 9:1920-36.

13. Emmott E, Smith C, Emmett SR, Dove BK, Hiscox JA: Elucidation of the avian nucleolar proteome by quantitative proteomics using SILAC and changes in cells infected with the coronavirus infectious bronchitis virus. Proteomics 2010, 10:3558-62.

14. Liu X, Ma H, Xu Q, Sun N, Han Z, Sun C, Guo H, Shao Y, Kong X, Liu S: Characterization of a recombinant coronavirus infectious bronchitis virus with distinct S1 subunits of spike and nucleocapsid genes and a 3' untranslated region. Vet Microbiol 2013, 162:429-36.

15. Liu SW, Zhang QX, Chen JD, Han ZX, Liu X, Feng L, Shao YH, Rong JG, Kong XG, Tong GZ: Genetic diversity of avian infectious bronchitis coronavirus strains isolated in China between 1995 and 2004. Arch Virol 2006, 151:1133-48.

16. Jones R, Ellis R, Cox W, Errington J, Fuller C, Irvine R, Wakeley P: Development and validation of RT-PCR tests for the detection and S1 genotyping of infectious bronchitis virus and other closely related gammacoronaviruses within clinical samples. Transbound Emerg Dis 2011, 58:411-20.

17. Cavanagh D: Coronavirus avian infectious bronchitis virus. Vet Res 2007, 38:281-97.

18. Kapczynski DR, Afonso CL, Miller PJ: Immune Responses of Poultry to Newcastle Disease Virus. Dev Comp Immunol 2013

19. Girardin SE, Sansonetti PJ, Philpott DJ: Intracellular vs extracellular recognition of pathogens-common concepts in mammals and flies. Trends Microbiol 2002, 10:193-9.

20. Bowie AG, Haga IR: The role of Toll-like receptors in the host response to viruses. Mol Immunol 2005, 42:859-67.

21. Okabayashi T, Kariwa H, Yokota S, Iki S, Indoh T, Yokosawa N, Takashima I, Tsutsumi H, Fujii N: Cytokine regulation in SARS coronavirus infection compared to other respiratory virus infections. J Med Virol 2006, 78:417-24.

22. Smith J, Sadeyen JR, Paton IR, Hocking PM, Salmon N, Fife M, Nair V, Burt DW Kaiser P: Systems analysis of immune responses in Marek's disease virusinfected chickens identifies a gene involved in susceptibility and highlights a possible novel pathogenicity mechanism. J Virol 2011, 85:11146-58.

23. Guo X, Wang L, Cui D, Ruan W, Liu F, Li H: Differential expression of the Toll-like receptor pathway and related genes of chicken bursa after experimental infection with infectious bursa disease virus. Arch Virol 2012, 157:2189-99

24. Nang NT, Lee JS, Song BM, Kang YM, Kim HS, Seo SH: Induction of inflammatory cytokines and toll-like receptors in chickens infected with avian H9N2 influenza virus. Vet Res 2011, 42:64

25. Li J, Liu Y, Zhang X: Murine coronavirus induces type I interferon in oligodendrocytes through recognition by RIG-I and MDA5. J Virol 2010, 84:6472-82.

26. Roth-Cross JK, Bender SJ, Weiss SR: Murine coronavirus mouse hepatitis virus is recognized by MDA5 and induces type I interferon in brain macrophages/microglia. J Virol 2008, 82:9829-38.

27. Yoshikawa T, Hill TE, Yoshikawa N, Popov VL, Galindo CL, Garner HR, Peters CJ, Tseng CT: Dynamic innate immune responses of human bronchial epithelial cells to severe acute respiratory syndrome-associated coronavirus infection. PLOS ONE 2010, 5:e8729.

28. Zhong Y, Tan YW, Liu DX: Recent progress in studies of arterivirus- and coronavirus-host interactions. Viruses 2012, 4:980-1010.

29. Pei J, Sekellick MJ, Marcus PI, Choi IS, Collisson EW: Chicken interferon type I inhibits infectious bronchitis virus replication and associated respiratory illness. J Interferon Cytokine Res 2001, 21:1071-7.

30. Holmes HC, Darbyshire JH: Induction of chicken interferon by avian infectious bronchitis virus. Res Vet Sci 1978, 25:178-81.

31. Otsuki K, Sakagami Y, Tsubokura M: Serological relationship among ten strains of avian infectious bronchitis virus. Acta Virol 1987, 31:138-45.

32. Bovolenta C, Lorini AL, Mantelli B, Camorali L, Novelli F, Biswas P, Poli G: A selective defect of IFN-gamma-but not of IFN-alpha-induced JAK/STAT pathway in a subset of U937 clones prevents the antiretroviral effect of IFN-gamma against HIV-1. J Immunol 1999, 162:323-30.

33. Haller $O$, Staeheli $P$, Kochs $G$ : Interferon-induced $M x$ proteins in antiviral host defense. Biochimie 2007, 89:812-8.

34. Sadler AJ, Williams BR: Interferon-inducible antiviral effectors. Nat Rev Immunol 2008, 8:559-68. 
35. Danesh $A$, Cameron $C M$, Leon $A J$, Ran $L, X u L$, Fang $Y$, Kelvin $A A$, Rowe $T$, Chen H, Guan Y, Jonsson CB, Cameron MJ, Kelvin DJ: Early gene expression events in ferrets in response to SARS coronavirus infection versus direct interferon-alpha2b stimulation. Virology 2011, 409:102-12.

36. Mogensen TH, Paludan SR: Molecular pathways in virus-induced cytokine production. Microbiol Mol Biol Rev 2001, 65:131-50

37. Huang KJ, Su IJ, Theron M, Wu YC, Lai SK, Liu CC, Lei HY: An interferongamma-related cytokine storm in SARS patients. J Med Virol 2005, 75:185-94.

38. To KF, Chan PK: Identification of human cell line model of persistent SARS coronavirus infection and studies of the response to cytokines and chemokines. Hong Kong Med 2009, J15(Suppl 6):39-43.

39. Zhang Y, Li J, Zhan Y, Wu L, Yu X, Zhang W, Ye L, Xu S, Sun R, Wang Y, Lou J: Analysis of serum cytokines in patients with severe acute respiratory syndrome. Infect Immun 2004, 72:4410-5.

40. Yasukawa H, Ohishi M, Mori H, Murakami M, Chinen T, Aki D, Hanada T, Takeda K, Akira S, Hoshijima M, Hirano T, Chien KR, Yoshimura A: IL-6 induces an anti-inflammatory response in the absence of SOCS3 in macrophages. Nat Immunol 2003, 4:551-6.

41. Cohen GM: Caspases: the executioners of apoptosis. Biochem J 1997, 326:1.

42. Zhong Y, Liao Y, Fang S, Tam JP, Liu DX: Up-regulation of Mcl-1 and Bak by coronavirus infection of human, avian and animal cells modulates apoptosis and viral replication. PLOS ONE 2012, 7:e30191.

43. Ding L, X X, Huang Y, Li Z, Zhang K, Chen G, Yu G, Wang Z, Li W, Tong D: Transmissible gastroenteritis virus infection induces apoptosis through FasL-and mitochondria-mediated pathways. Veterinary Microbiol 2012, 158:12-22.

44. Marfè G, Tafani M, Fiorito F, Pagnini U, lovane G, De Martino L: Involvement of FOXO transcription factors, TRAIL-FasL/Fas, and sirtuin proteins family in canine coronavirus type II-induced apoptosis. PLOS ONE 2011, 6:e27313.

45. Dunkelberger JR, Song WC: Complement and its role in innate and adaptive immune responses. Cell Res 2010, 20:34-50.

doi:10.1186/1471-2164-14-743

Cite this article as: Cong et al:: Transcriptome analysis of chicken kidney tissues following coronavirus avian infectious bronchitis virus infection. BMC Genomics 2013 14:743.

\section{Submit your next manuscript to BioMed Central and take full advantage of:}

- Convenient online submission

- Thorough peer review

- No space constraints or color figure charges

- Immediate publication on acceptance

- Inclusion in PubMed, CAS, Scopus and Google Scholar

- Research which is freely available for redistribution 